\title{
Vivir juntos: Acceso en 7 obras arquitectónicas de arte contemporáneo de París
}

\section{Living together: Access in 7 architectural works of contemporary art in Paris}

\author{
DOI: 10.17981/mod.arq.cuc.27.1.2021.08
}

Artículo. Fecha de Recepción: 27/05/2021. Fecha de Aceptación: 08/06/2021.

\author{
Laura Gallardo Frías ()
}

Universidad de Chile. Santiago (Chile)

lauragallardofrias@uchilefau.cl

\section{Paulina Román Manzo}

Universidad Miguel de Cervantes. Santiago (Chile)

proman@corp.umc.cl

Para citar este artículo:

Gallardo, L. y Román, P. (2021). Vivir juntos: Acceso en 7 obras arquitectónicas de arte contemporáneo de París. MODULO ARQUITECTURA CUC, $27,195-218,2021$. http://doi.org/10.17981/mod.arq.cuc.27.1.2021.08

\section{Resumen}

El acceso es clave en las obras arquitectónicas destinadas al arte contemporáneo. Para revisar el acceso, se propone una metodología basada en seis puntos: entorno, fachada, plano-suelo, plano-cielo, entrada y vestíbulo, donde, por medio de un corpus de imágenes y textos, se analizan siete obras de París: Pompidou, Fondation-Cartier, Le-Plateau, MACVAL, Centquatre, Palais-de-Tokyo y Fondation-Louis-Vuitton. Finalmente se sintetizan aspectos clave que pueden contribuir al análisis, proyectación y remodelación de obras arquitectónicas que cobijan arte contemporáneo: museos, centros de arte y centros culturales, entre otros. Así, se pone de manifiesto el potencial de este pequeño método de análisis que puede ser utilizado para otros casos de estudio, debido a la relevancia de los puntos propuestos en su vinculación con la ciudad, el barrio y visitantes, además de sus propias relaciones abiertas a distintas posibilidades que requieren de una constante reflexión e investigación que potencie su vivir juntos.

Palabras Clave: Arte Contemporáneo; Centro Cultural; Entrada; Fundación; Museo
Abstract

Access is a key in architectural works for contemporary art. To review the access, an analysis methodology based on six points is proposed: environment, façade, ground-plan, sky-plan, entrance and vestibule. In order to examine these points, seven architectural works from Paris are selected: Pompidou, FondationCartier, Le-Plateau, MACVAL, Centquatre, Palais-de-Tokyo and Fondation-Louis-Vuitton. The analysis is based on the six points, by means of a corpus of images and a textual corpus. Finally, the characteristics of these architectural works intersect through the established points, by synthesizing key aspects that can contribute to the analysis, projection and remodeling of architectural works that shelter contemporary art: museums, art centers and cultural centers, among others. Thus, the potential of this small analysis method that can be used for other case studies is highlighted, due to the relevance of the points proposed in their connection with the city, the neighborhood, the environment and its visitors, in addition to their own relationships, open to different possibilities that require constant reflection and research to enhance their living together.

Keywords: Contemporary Art; Cultural Center; Entry; Foundation; Museum 


\section{EL ACCESO}

Los museos son 'piezas motoras' para las ciudades (Layuno, 2003; Galland, 2014; Falcón, 2019; Gallardo, Toledo, Figueroa, Vera y Pérez, 2019), sin embargo, las obras arquitectónicas destinadas al arte contemporáneo, se hace referencia a museos, centros de arte y centros culturales entre otros, tienen una dificultad particular para atraer a los visitantes, por la propia definición del arte contemporáneo, como señala Badiou (2013):

El arte contemporáneo va a tomar, otra dirección, que estará ligada a los efectos que produce: el arte no será un espectáculo, ni una detención del tiempo, más bien será lo que compromete en el tiempo mismo y produce efectos en el tiempo (...), apunta hacia una acción que cuestiona y transforma al sujeto (p. 3).

Así, las obras arquitectónicas destinadas al arte contemporáneo pasan a ser 'aparatos' (Déotte, 2012) más de preguntas que de respuestas (Rispal, 2009), tensionando el vínculo con sus visitantes y, por tanto, con su entorno. Ante esta problemática emerge una pregunta central: ¿Qué elementos de una obra arquitectónica que cobija arte contemporáneo son clave en la vinculación de sus habitantes y entorno?

En una investigación multidisciplinar, desde los campos de historia, urbanismo, arquitectura, antropología, donde se analizaron cinco obras emblemáticas de arquitectura pública destinadas al arte contemporáneo, en la vinculación con sus visitantes y su contexto en Santiago de Chile, se definieron cinco elementos-clave: Conectividad; Legibilidad, "fácilmente identificable" (Lynch, 2006, p. 11); Accesibilidad; Recorridos y permanencias; y Flexibilidad. Esta investigación fue extendida a París para validar estos elementos y profundizar en uno de ellos.

Tras meses de observación, se llegó a la conclusión de que el acceso es una de las piezas fundamentales para las obras arquitectónicas que albergan arte contemporáneo, pues permite la conexión de los mundos exterior-interior, $y$, si bien es un elemento estático, es capaz de generar movimientos vinculados al: entrar, atravesar, transportar, descubrir, traspasar, entre otros. Se puede hablar de un 'ritual de paso' (Benjamin, 2005), es decir, una suerte de 'ceremonia' al pasar del mundo cotidiano a otro por descubrir y adentrase en otra temporalidad (Renault, 2000).

El acceso se vincula con el umbral que "ofrece a todos la posibilidad de entrar o regresar" (Genette, 1987, p. 8), abre la conexión intra-extra (Rancière, 2005), de un vaivén que se relaciona con conceptos como: límite, frontera, piel, pórtico, puerta, permeabilidad, porosidad, espesor, tensión, movimiento (visitante)-quietud (umbral) (Dorrian, 2014; Packer \& Ballantyne, 2002). 
El umbral funde los espacios (Bonnin, 2000). Así, "cruzar el umbral significa agregarse a un mundo nuevo. Es un acto importante" (Van Gennep, 1909, p. 27).

En la observación realizada, se puso de relevancia que un acceso sea identificable, accesible y seductor, es decir, que invite a entrar, y también que existen muchos tipos de accesos, pudiéndose realizar distintas taxonomías en función de su ubicación, nivel, tipo de superficie y delimitación, entre otras. Se llegó a la conclusión que el acceso va más allá de la puerta de entrada al museo, pues para llegar a él hay una serie de concatenación de elementos que entran en juego. Así, se sintetizaron seis elementos-clave, que permiten comparar distintos casos de estudio y sobre todo revisar cómo conviven con sus visitantes y entorno, que ordenados desde el afuera hacia el adentro son:

1. Entorno. Vinculación del museo con las construcciones vecinas en función a proporciones o escala, el volumen de la propia edificación, hasta los elementos que indican su presencia, como afiches y señales. En definitiva, cómo dialoga el museo con su barrio y desde dónde se aprecia.

2. Fachada. Características de la piel: opaca, transparente, semitransparente, color, textura, relación llenos-vacíos. ¿Cómo vincula el exterior-interior? ¿Qué elementos llaman la atención para invitar a entrar?
3. Plano del Suelo. Materialidades, texturas y colores asociados. ¿Cómo conduce hasta la entrada? si permite a la calle adentrarse al museo, o bien el museo sale hacia el exterior con su pavimento, produciéndose un interesante intercambio y llamada a acceder o jugar con sus límites.

4. Plano del Cielo. ¿Cómo es la parte superior que conforma el acceso? Generalmente se encuentran marquesinas o pórticos cuyo techo es relevante pues protege de la lluvia, indica dónde se encuentra la entrada y conduce a ella.

5. Entrada o umbral. La entrada es a la vez interioridad y afuera (Blanchot, 1992), pues conecta o separa ambos mundos (Gallardo, 2011). Revisión del tipo de puertas, altura, materialidad, color, etc.

6. Vestíbulo. ¿Cómo es el interior que puede o no asomarse hacia el afuera y cómo se establece esta relación?

Para revisar el acceso principal de visitantes a partir de estos elementos, se tomaron distintas obras arquitectónicas emblemáticas de arte contemporáneo de París (Francia). Los criterios de selección se basaron en considerar diferentes tipos arquitectónicos: centros culturales, fundaciones, museos y centros de arte; y obras de tamaños y escalas diferentes con distinta afluencia de público. Estas características se 
contrastaron in situ y en distintas páginas web, entre las que destacan: Office du Tourisme et des Congrès (2020), en particular el reportaje "santuarios del arte moderno y contemporáneo en París", el Observatoire de l'art contemporaine (2020) y el sitio del Ministère de la Culture (2020).

\section{ANÁLISIS DEL ACCESO EN SIETE OBRAS}

Se seleccionaron siete obras arquitectónicas que cobijan arte contemporáneo en París, ordenadas por año de inauguración son:

\section{Centre Georges Pompidou.}

El Centre Pompidou, con su estructura de cristal y metal atravesada por la luz, late en el centro de París como un corazón regado por arterias monumentales de colores primarios y brillantes (Centre Georges Pompidou, 2020).

\section{Fondation Cartier.}

Cada año, la Fondation Cartier programa exposiciones temáticas o personales y luego realiza pedidos con los artistas, enriqueciendo así una importante Colección (Fondation Cartier, 2020).

\section{Le Plateau.}

Cada año se organizan en Le Plateau cuatro exposiciones personales o temáticas que dan lugar a la producción de obras, incluida una realizada a partir de las colecciones (FRAC, 2020).

\section{Macval.}

El MAC VAL es el único museo dedicado exclusivamente a la escena artística en Francia desde la década de 1950 (MACVAL, 2020).

\section{Centquatre.}

El Centquatre es un espacio de residencias, producción y difusión para público y artistas de todo el mundo (Centquatre, 2020).

\section{Palais De Tokyo.}

Anclado en el presente y mirando hacia el futuro, el Palais de Tokyo también es rico de una historia fascinante, que lo invita a un viaje a través de la creación artística (Palais de Tokyo, 2020).

\section{Fondation Louis Vuitton.}

La Fundación ancla su apuesta por la creación actual desde una perspectiva histórica. En 2006, la creación de la Fundación Louis Vuitton marcó una nueva etapa en el patrocinio del grupo LVMH y sus casas (Fondation Louis Vuitton, 2020).

Para analizar estos casos, primero se presenta cada uno con una descripción: dirección, uso, arquitectos, inauguración, superficie, web y características principales del centro; después se introduce un corpus de imágenes basado en los seis puntos de análisis; y se describe cada punto. 


\section{Centre Georges Pompidou}

El Pompidou (Figura 1) está ubicado en la Place Georges-Pompidou, distrito-4. Desde su inicio fue una idea revolucionaria basada en la multidisciplinariedad cultural (Viatte, 2007). Cuenta con una biblioteca, un centro de investigación musical y acústica. Rompió con los paradigmas anteriores, no solo por sacar todas las instalaciones afuera, sino por abrirse a la plaza. El proyecto, ganador de un concurso internacional, fue diseñado por Renzo Piano y Richard Roger, se inauguró en 1977 y tiene 17000 m$^{2}$ cesibles a público (Centre Georges Pompidou, 2020).

El Centre Pompidou tiene la misma altura de Elificios vecinos, pero resalta los edificios vecinos, pero resalta en su entorno de virible esta difcacion des le los.

La fachan pran plano vidrado del que quedan a la vista la escalera mecánica insorta en un tubo transparente que le otorga un gran dinamismo, así como las

Lo más destacado del plano del suelo es la estrecha vinculación con la plaza inclinada del acceso principal, la cual pareciera descender hasta introducisse en el Centro. Cuando la plaza llega hasta el nivel de entrada, los adoquines rugosos se transforman en baldosas rectangulares lisas. Después, en el volumen situado debajo de la marquesina, el pavimiento se convierte en una alfombra hasta que pasa al
Modul. Arquit. CUC 27, 195-218, 2021

interior, donde el suelo es de hormigón a la vista, pero se marca cada auceso por una alfombra roja que direcciona al visitante hasta el vestíbulo. En el plano del cielo una gran marquesina vidriada sobresale de la fachada para marcar el acceso principal. Debajo de esta marquesina existe una banda horizontal de letras que van anunciando distintas informaciones y un volumen vidriado, donde se sitúa el control de seguridad, con un falso cielo de un material reflectante blanco que conecta con el cielo interior, con tubos azules a la vista, que introduce al visitante en el vestíbulo de gran altura.

El acceso se conforma por un volumen ubicado bajo la marquesina que sobresale de la fachada. Las puertas exteriores tienen una proporción de una persona y media aproximadamente, de tal forma que, al disminuir la altura, tras las grandes proporciones de la fachada y la plaza, se produce una suerte de 'juego de escalas' al secercar a la escala humana en el ámbito del control de a la eriad, y después volver a elevar fuentrol de la altura en el vestíbulo. Al interior, las puertes quedan en el mismo plano de la fachada. quedan en el mismo plano de la fachada.
El vestíbulo, de gran altura, es un amplio espacio que funciona como una plaza con lugares También de exposición, con una tienda y una librería.

Figura 1. Centre Pompidou (Análisis del acceso). Fuente: Elaboración propia, 2019 [Archivo personal]. 
"Espacio de creación por los artistas y lugar de reencuentros entre el arte y el gran público", se define la Fondation Cartier (Figura 2) en su web (Fondation Cartier 2020, párr. 3). Diseñada por Jean Nouvel, a partir de un juego de trana por Jean Nouvl, a partir de pan jue de transparencias y restevas Raspail, no 261, del distrito-14. Situada Situada en cuenta con una superficie de $1200 \mathrm{~m}^{2}$ de salas de exposiciones, seis pisos de oficinas y su inau-
guración fue en 1994 .
Desde lejos esta fundación se mimetiza con

fundación se mimetiza con su entorno por el vínculo que posee con los árboles. Si bien la escala es acorde con los edificios aledaños, su piel comienza a destacar cuando el visitante se aproxima, ya que algo distinto se aprecia desde cerca.

En la llegada se descubre una fachada muy interesante por su piel transparente que juega con los límites del edificio y con la relación interior-exterior, tanto por sus aberturas, por las que se asoman los árboles, como por el juego de reflejos donde se entrecruzan adentro-afuera. Existe una doble fachada, ambas vidriadas, por un lado, la que limita al edificio con la calle que configuraria la primera entrada, y una segunda que, tras atravesar un patio, conduce al vestíbulo.

La aproximación se realiza por una vereda estrecha en la que no se encuentra en el plano del suelo ningún elemento que indique la presencia del Centro. El juego de reflejos de la

fachada es tan fuerte que conduce la mirada hacia un plano medio. En la primera entrada, existe un pequeño escalón metálico que conduce hasta el patio. En el patio se encuentra una especie de alfombra de baldosas que dirigen los visitantes al segundo acceso y desde allí al interior, donde continúan estas baldosas hacia el vestíbulo.

No existe plano del cielo en la entrada. Tras cruzar este primer umbral se pasa directamente al patio abierto donde el plano del cielo se construye con las copas de los árboles. En la segunda entrada el plano del cielo está conformado por una pequeña moldura metálica exterior y al interior un plano blanco, ubicado entre dobles puertas, conduce hasta el vestíbulo con la misma altura.

La entrada principal es simplemente un corte entre los planos vidriados de la fachada que con una reja metálica configuran un vacío para el acceso. Tras atravesar el patio se llega al segundo acceso que se marca con puertas correderas automáticas de vidrio encima de las cuederas autosa una abundante vegetación en eles sode fachada.

En el vestíbulo, - atravesado en su centro por unas escaleras que conducen a la sala inferior de exposiciones-, llama la atención su escasa altura, la cual potencia la gran altura de las salas contiguas de exposición 
baja de un edifica de vivien de planta baja de un en del distrito-19. Si bien es el centro más pequeño con $600 \mathrm{~m}^{2}$ fue seleccionado por su integración con 600 mario y por ser muy importante para los con el bary y por sor artistas parilizaron para la creacin de este la Socie (SFP) de Belleville. Está Prodich (SFP) de Belleville. Está enfocado en la creación, Fue Fue encargado por en Contenporán por Jean-Marc La

Es un caso interesante por su ubicación en la planta baja de un edificio de viviendas, que dialoga muy bien con su entorno pasando desapercibido, a no ser por los pequeños letreros que marcan su presencia.

En la fachada predomina lo sólido por sobre los vacíos, con una modulación sencilla de placas rectangulares gris claro en la planta baja que continua en toda la edificación en un tono más claro.

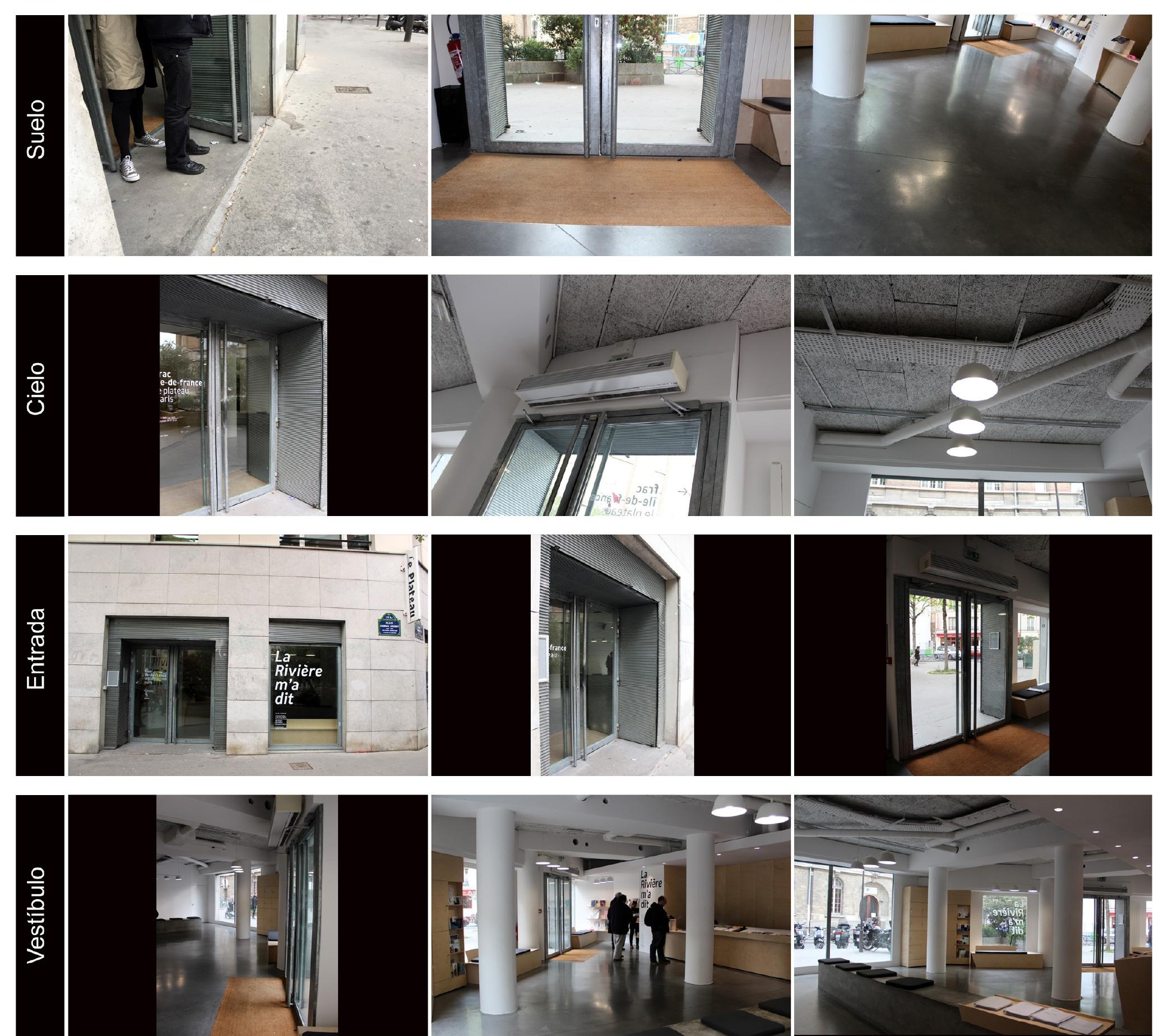

El plano del suelo se mimetiza totalmente, pues es el mismo material de hormigón del exterior que se desliza al interior con un mínimo escalón, y después, tras pasar por una alfombra, se transforma en hormigón pulido que permite reflejar la luz otorgándole una mayor luminosidad al vestíbulo.

El plano del cielo no sobresale, sino que se encuentra tragado en el dintel y conformado con la misma superficie metálica ondulada que reviste el umbral, marcando de esta forma la entrada. En el interior, el cielo está compuesto por paneles acústicos e instalaciones a la vista pintadas de blanco.

La entrada es muy sobria, está conformada por una doble puerta vidriada enmarcada po una superficie metálica ondulada, rehundida con respecto al plano de la fachada exterior. El vestíbulo es de tamaño mediano muy luminoso, de escasa altura, cuenta con una pequeña recepción, libreria y lugares para sentarse. 
El Musée d'art Contemporain du-Val-de-Marne (MACVAL) (Figura 4) se ubica en la Place de la Libération, Vitry-sur-Seine Nace con el objetivo Liberation, Viterive Name al arte contempo ráneo, con un programa de: salas de exposición talleres pedagóficos, talleres de artistas, auditó taller rium, cafti proyect 11000 muhart, cuenta con $11000 \mathrm{~m}^{2}$ y se inauguró
(Jacques Ripault Arquitecture, 2005).

Al transitar por la vereda se observa una edificación distinta por su geometría y por la relación de sus llenos y vacíos, ya que está conformada por grandes planos de hormigón armado con una ventana longitudinal. Al llegar a la esquina, el MACVAL se retira para conformar una plaza compuesta de árboles, bicicleteros y bancos que compuesta de árboles, bicicleteros y bancos que como antesala. Así, el museo además ye dialogar con el barrio, por su escala similar, lo potencia con este lugar de reunión que abre la posibilida con este la de susibilidad te con su nombre.

laterales opacas conducen hasta la fachada principal ubicada en la plaza, que está compuesta por un volumen opaco, situado en la parte izquierda que sobresale hacia la plaza, y un zócalo vidriado que marca el acceso y conecta el museo con el espacio público.
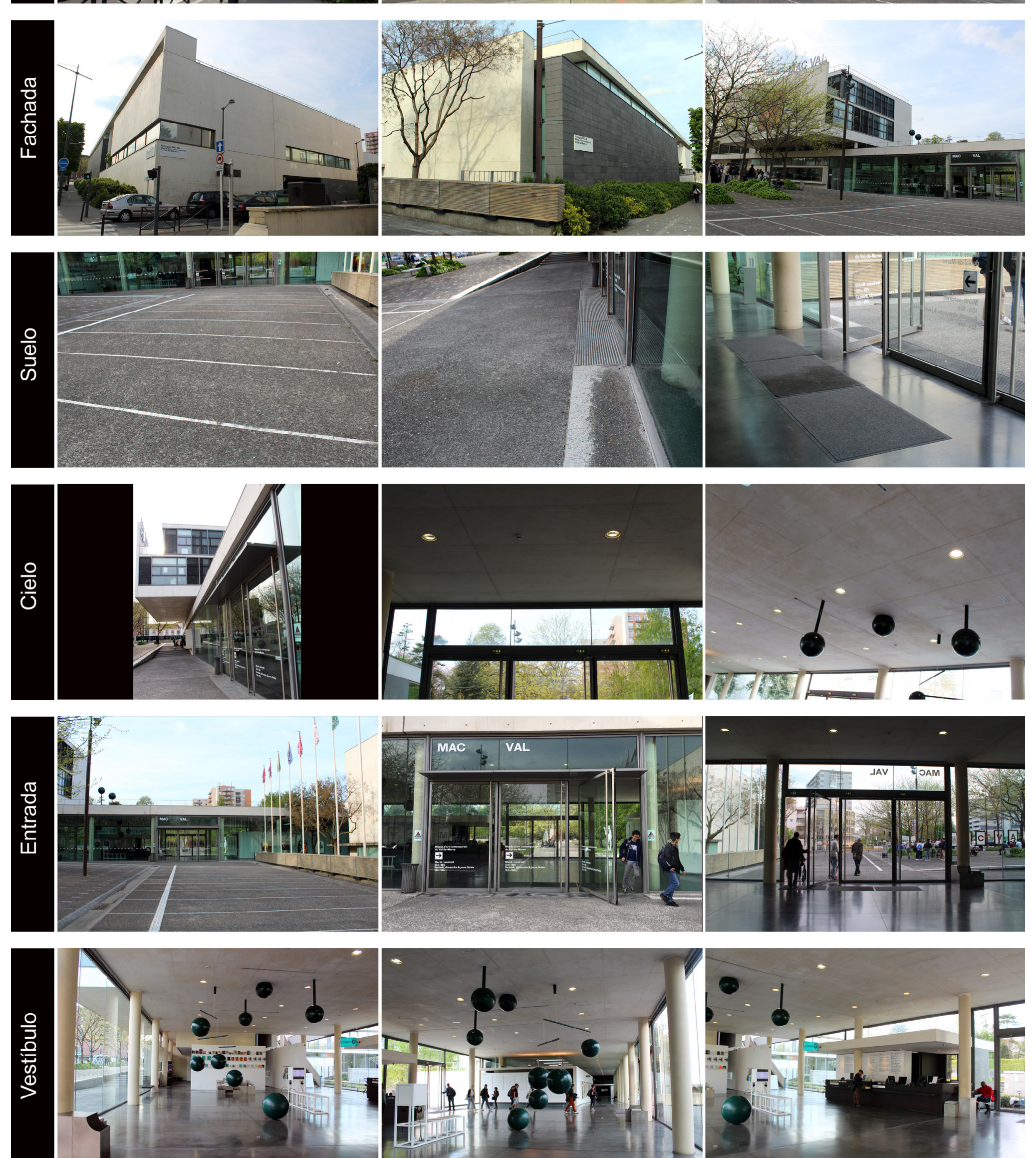

puesto por un gran tapiz de hormigon granulado gris oscuro que va jugando con piezas blancas. En el tramo del acceso, las líneas blancas permiten direccionar al visitante hasta la puerta principal. Antes de entrar hay una pequeña franja en la que se ubica una alfombra metálica con bandas antideslizantes que conduce hasta el interior, donde el plano del suelo conserva la misma tonalidad y nivel que el exterior, pero se transforma en hormigón pulido.

El edificio, al retirarse para conformar la plaza, permite que el cielo se haga presente. El plano del cielo exterior del edificio está conformado por una pequeña marquesina que indica el acceso. Al entrar hay un ligero cambio de altura y el plano del cielo del vestíbulo, de unos cinco metros de altura aproximadamente, se conforma por una gran superficie de hormigón armado color claro.

La entrada, direccionada además del plano del suelo por un plano vertical donde se ubicano del deras, está conformada por tres puertas de vidrio decilobatientes, de altura aproximada de cudrio metros, integradas en el plano de fachada vidriado. El vestíbulo cuenta con una amplia superficie cher vite cour con los demás ámito que perite cur porales y una librería.

Figura 4. MACVAL (Análisis del acceso). Fuente: Elaboración propia, 2019 [Archivo personal]. 


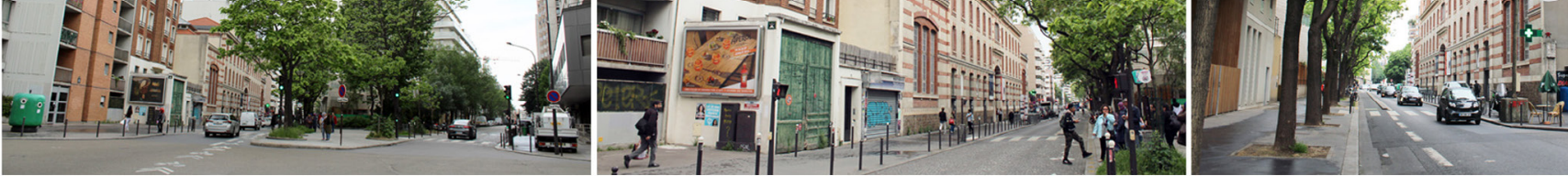

El Centquatre (Figura 5) ubicado en la calle d'Aubervilliers, no. 104, del distrito-19, era originalmente una antigua funeraria proyectada por Edouard Delebarre Debay y Godon, la cual pue recortida en foveconve: "Un lugar infinito de at te, cutteler (Centura e 2020). Pen" con 2020). Pen codo laborativa que da acon una programación contenporánea y un extenso

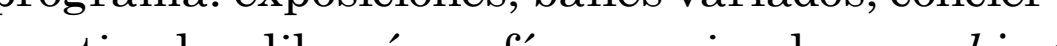
tos, tiendas, libreria, cafes, espacios de coworking y arriendo de salas, entre otros muchos. Tiene $35000 \mathrm{~m}^{2} \mathrm{y}$ fue inauguado on co caso que no fue diseñado originalmente para ser centro cultural, pero se subraya su gran exito marcado por su elevado número de visitantes. Si bien en el barrio hay edificaciones de distintas alturas, el 104 se integra perfectamente $e$ ncluso desde lejos cuesta identificarlo. Se sitúa en una vereda estrecha de una calle con una vía de circulación, aunque de bastante tránsito, donde resaltan los árboles de la vereda del frente.

La fachada es antigua dentro de un contexto de edificios nuevos y se destaca el ritmo de llenos y vacíos. Existe una secuencia en el acceso conformada por dos fachadas separadas por un patio. La fachada segunda sigue con la modulación y materialidad exterior, sin embargo, se compone de un gran plano vidriado coronado por un arco de medio punto que amplía la condición y vocación del acceso inicial.
El trabajo de suelo resulta muy interesante por su sutileza, pues los adoquines salen hacia el exterior y toman el color gris del asfalto de la vereda marcando y llamando fuertemente hacia el interior. Al traspasar el umbral, estos adoquines se tornan de distintos colores de tonalidades ocres y al pasar por la segunda fachada hay un cambio de pavimento, que al interior se transforma en hormigón pulido.

No existe ningún elemento en el plano del cielo que sobresalga para marcar la entrada, simplemente se indica a partir de la bóveda conformada por la boca del acceso al centro. Tras este primer plano superior, se pasa al cielo abierto en el patio que conecta ambas fachadas del acceso. $Y$ en la segunda fachada, una viga de madera laminada, situada a doble altura de la puerta, marca los accesos laterales.

La entrada, si bien tiene dos accesos se describe el principal, que le da nombre al centro, se indica con una gran abertura en la fachada conformada por una bóveda con una altura aproximada de tres veces una persona, el cierre se realiza medieste una reja abierta ducte día. Esta idea de gran váí se leva al segundo acceso, donde existe un

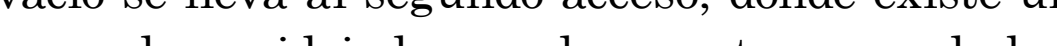
mana, que conectan con el interior.

El veś́bulo es muy reducido siendo su principal ín funcion información.

Figura 5. Centquatre (Análisis del acceso). Fuente: Elaboración propia, 2019 [Archivo personal] 


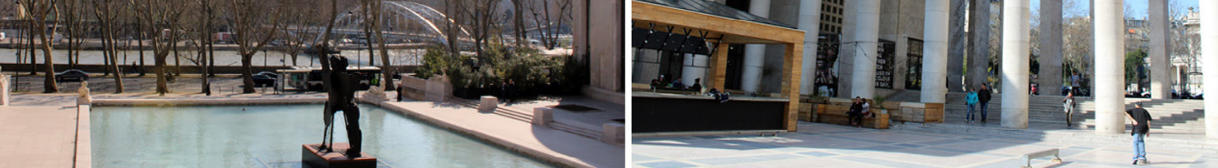

En el mismo edificio se encuentran: en el ala este el Museo de Arte Moderno de París y en el ala oeste el Palais de Tokyo (Figura 6), ubicado en el número 13 de la Avenue du Président Wilson, del distrito-16. Se inaugura en 1937 para la Exposición Internacional de París como Palais des Musées d'art moderne, desde entonces siempre ha albergado programas creativos vinculados pre ha alberado Lacaton \& Jean-Philippe Vassal con el nombre de Site de Jétion contemporaine, esecializre de Site de creation tico en el pancrama an ternacion. Adenás de las a desión y En 2012 estos mi En el 2012 estos ron paracor es centro de artectenter

El Palais de Tokyo dialoga con los edificios vecinos con su altura y estilo similar. Lo más representativo es la conexión entre los museos de Arte Moderno y Palais de Tokyo a través de un pórtico doble, conformado por altísimos pilares, que logra conectar con la calle paralela abriendo la vista hacia la torre Eiffel, el Sena y la plaza que se conforma al atravesar dicho pórtico situada entre los dos museos, que con un plano de agua en su centro también consigue la conexión con el cielo.

La fachada se concibe como una totalidad compuesta por las dos alas de los museos y el pórtico que las conecta. La fachada que da al Sena se abre a través de distintos espacios públicos, situados en diferentes niveles entre los dos museos. El plano del suelo es complejo porque se observan numerosos escalones, pero también existe una pequeña rampa metálica para las personas con movilidad reducida hacia otro acceso lateral. Hacia el asfalto de la vereda, salen unos escalones de mármol con forma redondeada que marcan la entrada al Palais de Tokyo y lo conectan con el vestíbulo.

El plano del cielo se sitúa en un marco estrecho y alto que perfila el acceso. Está conformado por un dintel de piedra blanca que sobresale ligeramente con respecto al plano de la fachada, y se entrecruza con el cielo curvo, con la misma altura del primer ámbito del vestíbulo.

La entrada está enmarcada por un rectángulo en fachada dibujado con el dintel y una ranura que marca la verticalidad en los laterales, junto con unas jambas metálicas tragadas que acentúan su esbeltez. Tras la apertura de la piel, se encuentra un plano de vidrio curvo, en toda la altura de la abertura de la fachada, que contiene dos puertas vidriad para comunicar con el pidos puertas vidriadas para

El vestíbulo no se advierte desde el exterior y Ex se compone tico nón lu de dén, un espacio para sentarse y las coxicepcon salas, librería y café.

Figura 6. Palais de Tokyo (Análisis del acceso). Fuente: Elaboración propia, 2019 [Archivo personal]. 


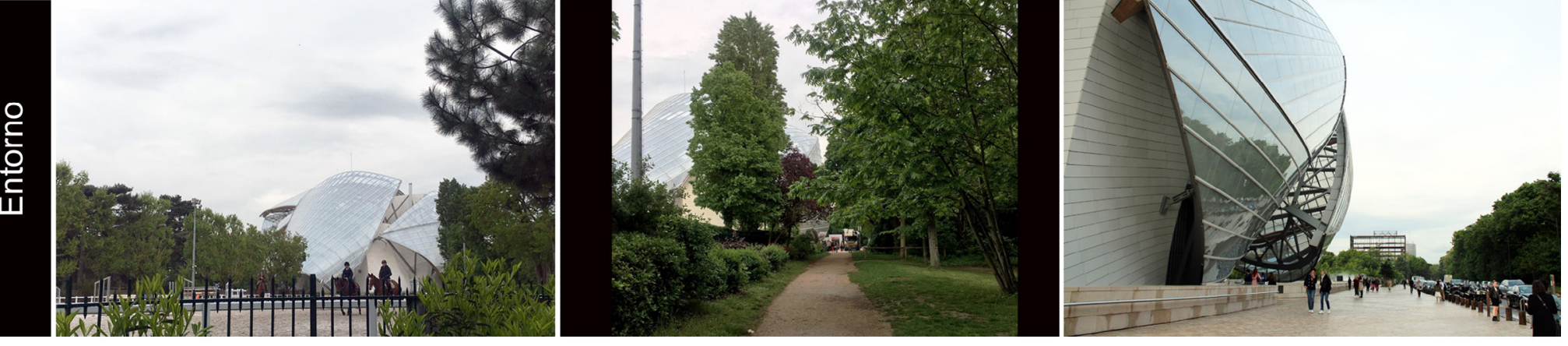

Fondation Louis Vuitton

La Fondation Louis Vuitton-LV (Figura 7) se encuentra en la 8 Avenida del Mahatma Gandhi, del distrito-16, en pleno bosque hi, ne. Prosecto de de arte collos de arte con Tiene una supefice tot de $11000 \mathrm{~m}^{2}$, de los 7000 esta abiertos al pílico, y fue ina que 7000 sstann abictos al público, y fue inaugurado en el 2014 (10 2020, Gehry, 2015).

Vuitton resalta con resdonde se inserta por su volumetría. La fachada es asombrosa, tanto por su altura como por los planos de vidrio curvos que la conforman, emergiendo como un hito distinto y llamativo.

El plano del suelo de la vereda pasa del asfalto a convertirse en adoquines en todo el perímetro de la fachada principal. Los adoquines se transforman en adoquines de mayor tamaño en el acceso y estos a su vez en baldosas de mayor tamaño en el límite de la edificación para marcar la entrada, todo en tonalidad ocre.
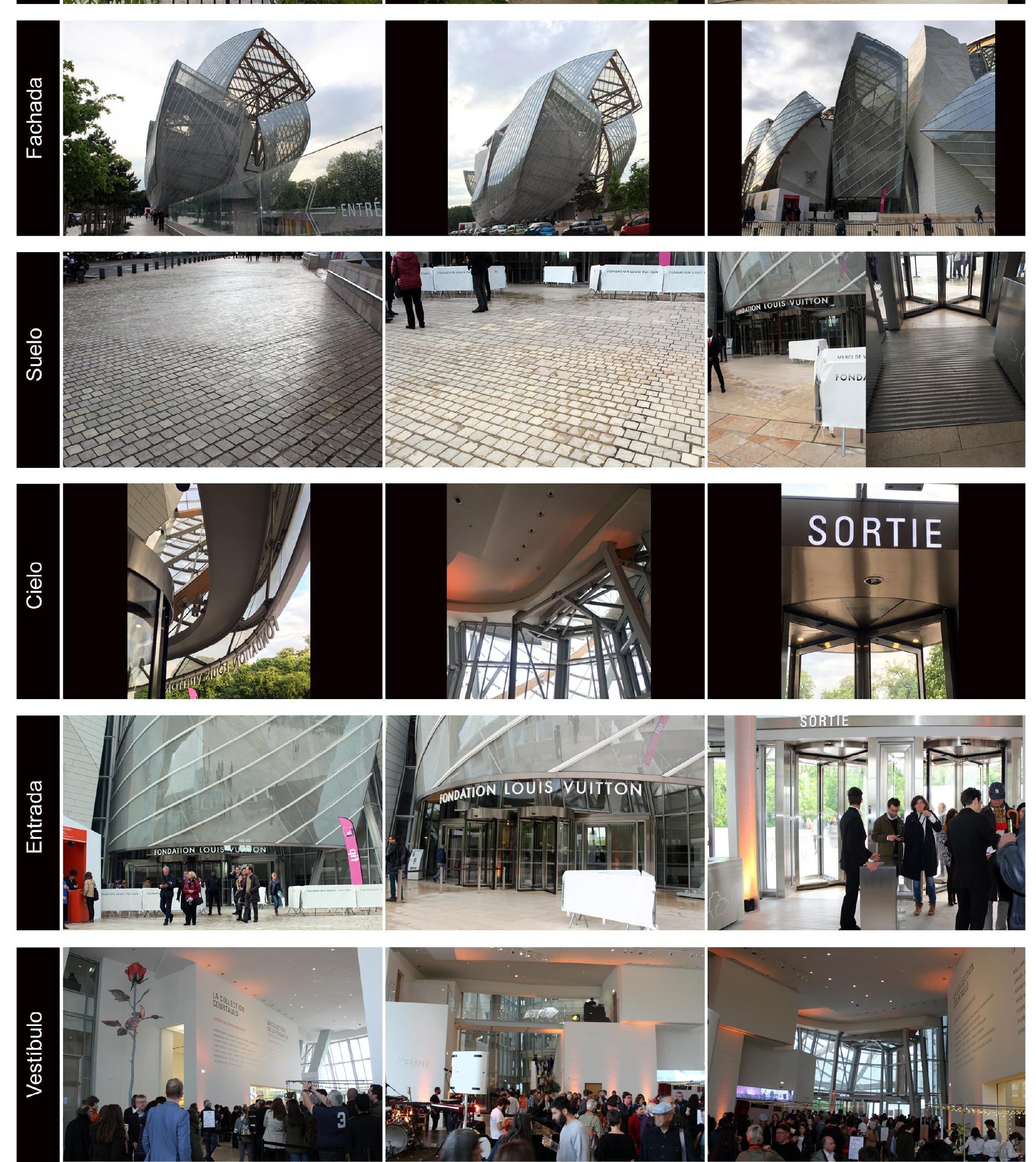

El plano del cielo está conformado por una viga metálica curva que permite marcar sutilmente el acceso principal vinculándolo con el volumen curvo superior de la fachada. Al interior, antes de atravesar la puerta, otra viga metálica curva marca el espacio vacio entre la piel de la fachada vidriada y las puertas de acceso. Después, en las puertas giratorias el plano del cielo se convierte en una superficie metálica pulida. En el vestíbulo, el cielo se eleva en cuatro veces, aproximadamente, la altura de una persona y se convierte en un plano blanco liso, donde se insertan distintas luces.

Antes de acceder, el personal de seguridad revisa las pertenencias de los visitantes en una caseta ubicada en el lateral del museo. La entrada está conformada por dos puertas giratorias y puertas correderas a ambos lados, todas vidriadas y situadas debajo del volumen curvo de la fachada que marca el acceso con el nombre del museo suspendido de una viga metálica.

El vestíbulo es muy amplio y permite que se realicen simultáneamente distintas actividades: informaciones, cafetería, a veces música en vivo con DJ. También permite conectar los distintos programas que posee esta fundación: restaurante, salas de exposiciones, librería, auditorio, talleres, entre otros. 


\section{Vivir juntos}

El análisis realizado ha permitido recorrer, identificar y examinar, los elementos-clave que conforman el acceso. Por tanto, este pequeño método se podría utilizar en otros casos de estudio considerando que la debilidad de cualquier método es no mirar más allá de los puntos establecidos y por supuesto tener presente que siempre es mejorable. Se revisan a continuación los seis puntos estudiados.

El Pompidou se distingue como un hito en la ciudad, característica que comparte con la Fondation Louis Vuitton. Desde su entorno cercano, se distinguen otros puntos-clave: que sea legible e identificable, desde lejos y desde el propio barrio (Fondation Cartier y Palais de Tokyo); que se mimetice con su entorno (104 y le Plateau); que tenga una estrecha vinculación con el espacio público (Pompidou); y que conforme una antesala para sacar el arte a la calle y abrir la experiencia con la comunidad (MACVAL), donde también se valoran los letreros que indican la presencia del Centro (Le Plateau).

La vinculación con el entorno tiene una relación directa con la fachada, donde emerge la característica de 'piel sugerente', recomendable para denotar la presencia e invitación a entrar, como en la Fondation Cartier, Louis Vuitton, Palais de Tokyo y Pompidou. Este último Centro también tiene una cualidad remarcable que radica en la transparencia de su fachada que permite, al igual que el MACVAL, percibir desde afuera el interior. Así, se pueden considerar distintos grados de porosidad de la piel, en un grado menor en Le Plateau, donde a través de aberturas más pequeñas se deja entrever lo que sucede en el interior, y en uno mayor está la gran abertura que muestra el 104 para acceder.

El plano del suelo juega un papel fundamental, se destaca la accesibilidad universal presente en todos los casos (De la Iglesia-Mayol y RosellóRamón, 2014; Fernández, 2013), y los distintos tratamientos para la continuidad exterior-interior que se observa en cinco de los centros estudiados. En el caso emblemático del Pompidou, el exterior se introduce en el interior, en la Fondation Louis Vuitton, MACVAL y en Le Plateau, se producen cambios sutiles con materiales, texturas, formatos y colores, jugando con la continuidad dentro-fuera, y en el 104 el pavimento sale hacia el exterior. Solamente en la Fondation Cartier y en el Palais de Tokyo no sucede esta continuidad, en el primer caso se suple por la maravillosa piel de vidrio que hace que se pierda de vista el plano del suelo, y en el segundo caso, la escalera se despliega hacia el exterior haciendo un llamado al acceso.

$\mathrm{Al}$ contrario de lo que se podría pensar para este tipo de edificios, solamente el Pompidou tiene una gran marquesina, que ayuda a conformar el umbral, el MACVAL tiene otra, pero de escasas dimensiones. La mayoría de los casos presentan el dintel como elemento representativo del plano del cielo para marcar el acceso: Le Plateau, Le Palais de Tokyo, 104 y la Fondation LV, esta última con su nombre suspendido. En una tercera alternativa se encuentra la Fondation Cartier con una abertura completa en la fachada, teniendo 
al propio cielo como plano de acceso. Se destaca este hallazgo de llegar a enmarcar o 'capturar' el cielo, - como sucede en 104, Fondation Cartier y Palais de Tokyo-, que consigue un vínculo muy especial, pues abre y conecta con la dimensión espiritual otorgando aire y luz.

En el umbral es importante revisar las tres dimensiones. La altura en la primera puerta de entrada, es interesante destacar la relevancia de la primera entrada que era denominada Ianua en honor a Jano a quien se le consagraban todas las entradas y salidas (De Sevilla, 2004), la cual, si bien marca el acceso a un edificio emblemático, es clave que su dimensión sea ajustada al visitante, quizás con la intención de envolverlo o arroparlo, como lo demuestran todos los casos observados excepto el 104, donde hay que recordar es el único caso que no fue proyectado para albergar arte. Este énfasis en la experiencia 'solitaria' del acceso también se evidencia en el ancho de las puertas de una persona, que se da en todos los casos estudiados, excepto el 104. En la profundidad del umbral hay que distinguir entre espesor y dilatación (Espinosa, 2012), vacío y acumulación de capas, respectivamente, ambos relacionados con la distancia, el deseo de alargar el ingreso. Esto se consigue de distintas formas: en el $104 \mathrm{y}$ en el Pompidou esta distancia se vincula al espesor del acceso; en la Fondation Cartier se alcanza con el patio entre los accesos; en el Palais de Tokyo hay una pequeña antesala circular, ubicada antes del hall, que alarga la experiencia; y en Le Plateau el ancho de las jambas le otorgan un cierto espesor al acceso.
Con relación a las puertas, Quetglas (2004) llega a la conclusión que, para tener una suerte de ritual de acceso, deberían abrir hacia ambos lados, esto solo está presente en el MACVAL, el resto de los Centros tienen sus puertas principales abiertas, excepto Le Plateau y Le Palais de Tokyo que se abren hacia afuera y la Fondation LV que tiene puertas giratorias.

En el umbral las ideas luz y límites están estrechamente unidas, "umbral es borde de luz" (Gausa et al., 2001, p. 599). En los casos analizados emerge un hallazgo relevante referente a la luz en el umbral, con relación a la materialidad de las puertas de entrada, pues todas son de vidrio, excepto en el 104 y la Fondation Cartier, donde no existen puertas en el acceso. Esto hace destacar la importancia de la transparencia total en el umbral de los centros de arte y museos para tener claridad absoluta en el vínculo exteriorinterior.

En el vestíbulo, en todos los casos, salvo en las fundaciones Cartier y LV, el plano del suelo es de hormigón pulido y funciona muy bien, pues al ser reflectante aumenta la luminosidad, conserva la limpieza y se percibe como una superficie continua para deslizarse en ella sin distracciones y conectar con otros ámbitos y niveles. La mayoría de los vestíbulos funcionan como plazas con amplios espacios en los que los visitantes pueden sentarse a conversar, leer, revisar internet, incluso hacer fiestas. Solamente en el 104 y la Fondation Cartier, el vestíbulo es más reducido, donde se concentra el punto de informaciones y la distribución hacia el resto de los recintos. 
Además de los puntos estudiados, también hay que tnere en cuenta como elementos que invitan a entrar: las diferentes actividades, programas, el tipo y la calidad de las obras expuestas (Bourdieu y Darbel, 2004) y el juego de distintas escalas, tan considerado por Alvar Aalto (GarcíaEscudero \& Bardí, 2014), que generalmente continúa al interior del centro.

Se llega a la conclusión, tras la observación y el análisis realizado, que el acceso es esencial en las obras arquitectónicas que cobijan arte contemporáneo, el cual se compone de elementosfundamentales vinculados estrechamente para que funcionen como un auténtico engranaje con la finalidad de vivir juntos. Este es un conceptoclave ya que, como muestra Platón (1988), vivir juntos es olvidar las sombras para llegar a lo esencial, considerando que la ciudad no es solo una comunidad de lugar, sino que sustenta la elección de vivir juntos como finalidad (Jullien, 2018).

Vivir juntos refiere en este contexto, al vínculo entre la ciudad, el barrio, los elementos estudiados y sus posibilidades. Vivir juntos entrelazando la importancia de prever y también la de reflexionar, teniendo al ser humano como centro, para construir vínculos más estrechos (Touraine, 2000).

"Vivir-Juntos implica las huellas activas de la escucha" (Barthes, 2005, p. 131), es decir, conocer muy bien en el acceso: el entorno, la fachada, el plano del suelo, el plano del cielo, el umbral y el vestíbulo; elementos que trascienden como simples conectores y darles continuidad (Aalto, 2000), a la hora de analizar, proyectar o remodelar obras arquitectónicas que cobijan arte contemporáneo, como museos, centros de arte y centros culturales, entre otros. En definitiva, se pone de manifiesto la necesidad de seguir reflexionando sobre el acceso, la vinculación de estos centros y museos con su entorno y sus visitantes, y de una constante investigación que potencie su coexistencia, su vivir juntos.

\section{Agradecimientos}

A François Mairesse, presidente del Comité International pour la Muséologie de l'ICOM (ICOFOM), en la acogida de la estadía de investigación durante el 2019 en la Université Sorbonne-nouvelle (Paris, France).

A todo el equipo de investigadores del proyecto: María Isabel Toledo Jofré (Dra. Ciencias de la Educación), Consuelo Figueroa Garavagno (Dra. (c) Historia), Javier Vera Bravo (Antropólogo) y nuestro ayudante Luís René Pérez Huenupi (Arquitecto).

\section{REFERENCIAS}

Aalto, A. (2000). De los escalones de entrada a la sala de estar. En, G. Schildt (Ed.), Alvar Aalto de palabra y por escrito (pp. 69-74). Madrid: El Croquis Editorial.

Badiou, A. (mayo 11, 2013). Las condiciones del Arte Contemporáneo. Esfera pública. [publicación en blog]. Disponible en https:// 
esferapublica.org/nfblog/las-condiciones-delarte-contemporaneo/

Barthes, R. (2005). Cómo vivir juntos. Notas de cursos y seminarios en el Collège de France 1976-1976. Buenos Aires: Siglo XXI.

Benjamin, W. (2005). Libro de los pasajes. Madrid: Akal.

Blanchot. M. (1992). El espacio literario. Barcelona: Paidós.

Bonnin, P. (2000). Dispositifs et rituels du seuil. Communications, Seuils, passages, (70), 6592. https://doi.org/10.3406/comm.2000.2064

Bourdieu, P. y Darbel, A. (2004). El amor al arte: los museos europeos y su público. Buenos Aires: Paidós.

Centquatre. (2020). 104. [Online]. Disponible en https://www.104.fr/

Centre Georges Pompidou. (2020). ¿Quiénes somos? centrepompidou.fr/. Disponible en https://www.centrepompidou.fr/es/

De la Iglesia-Mayol, B. y Rosselló-Ramon, M. (2014). Identificación de las barreras de acceso a la información, a la participación y al aprendizaje en el Museo Es Baluard. Arte, Individuo y Sociedad, 26(1), 21-38. https://doi.org/10.5209/rev_ARIS.2014.v26. n1.40147

De Sevilla, I. (2004). Etimologías. Madrid: BAC.

Déotte, J.-L. (2012). ¿Qué es un aparato estético? Benjamin, Lyotard, Rancière. Santiago de Chile: Metales Pesados.

Dorrian, M. (2014). Museum atmospheres: notes on aura, distance and affect. The Journal of Architecture, 19(2). 187-201. https://doi.org/ 10.1080/13602365.2014.913257
Espinosa, J. (2012). El umbral como arquitectura. Espacio y transición en el límite de la casa. Revista de historia y teoría de la arquitectura, 10-11(2), 133-170.

Falcón, J. M. (2019). La arquitectura del museo: testigo y evidencia de la época. Arquiteturarevista, 8(2), 135-147. https://doi.org/10.4013/ arq.2012.82.04

Fernández, M. T. (2013). Los museos accesibles en España: el caso Guggenheim. Pasos 11(2), 399-415. https://doi.org/10.25145/j. pasos.2013.11.026

Fondation Cartier. (2020). History and missions. fondationcartier. Disponible en https://www. fondationcartier.com/?locale $=\mathrm{fr}$

Fondation Louis Vuitton. (2020). fondationlouisvuitton. [Online]. Recuperado de: https:// www.fondationlouisvuitton.fr/fr.html

FRAC. (2020). fraciledefrance. [Online]. Disponible en https://www.fraciledefrance. com/

Galland, D. (2014). Procesos y estilos de planificación en la rehabilitación urbana: el caso de Dinamarca. Revista de Arquitectura, 19(27), 15-24. Disponible en https://dearquitectura. uchile.cl/index.php/RA/article/view/33564

Gallardo, L. (2011). Vínculo interior-exterior. Una reflexión sobre la arquitectura el Lugar y el No-Lugar'. Revista 180, (27), 2-5. Disponible en http://www.revista180.udp.cl/ index.php/revista180/article/view/119

Gallardo, L., Toledo, M., Figueroa, C., Vera, J., y Pérez, L. (2019). Centro Nacional Arte Contemporáneo Cerrillos (CNACC): motor de transformación de la ciudad de Santiago. 
Revista de Urbanismo, (40), 1-19. Disponible en https://revistaurbanismo.uchile.cl/index. php/RU/article/view/52362

García-Escudero, D. y Bardí , B. (2014). El recorrido como estrategia en Alvar Aalto Análisis de cuatro obras, 1926-28. VLC arquitectura Research Journal. 1(1), 1-30. http://dx.doi. org/10.4995/vlc.2014.1888

Gausa, M., Guallart, V., Müller, W., Soriano, F., Porras, F. y Morales, J. (2001). Diccionario Metápolis de arquitectura avanzada. Barcelona: Actar.

Genette, G. (1987). Seuils. Paris: Seuil.

Gehry, F. (nov. 3, 2015). Fondation Louis Vuitton. arqa. Disponible en https://arqa.com/ arquitectura/fondation-louis-vuitton.html

Jacques Ripault Arquitecture. (2005). Musée d'art Contemporain MACVAL á Vitry Sur Seine. [Online]. Disponible en https://www.jacquesripault.com/ripault/references/culturel/ musees/mus\%C3\%A9e-d\%E2\%80\%99artcontemporain-macval-\%C3\%A0-vitry-surseine/

Jullien, F. (enero, 2018). La identidad cultural no existe. [publicación blog]. Disponible en http:// blogdejoaquinrabassa.blogspot.com/2018/01/ francois-jullien-la-identidad-cultural.html

Lalo, J-M. (2020). Le Plateau. [Online]. Recuperado de: https://www.atelierlalo.com/projets/ le-plateau/

Layuno, M. Á. (2003). Museos de arte contemporáneo y ciudad. Los límites del objeto arquitectónico. En: Lorente, J.P. y Almazán, D. (Cord.), Museología crítica y arte contem- poráneo (pp. 109-123). Zaragoza: Prensas Universitarias.

Lynch, K. (2006). La imagen de la ciudad. Barcelona: Gustavo Gili.

MAC VAL. (2020). VAL de MARNE. [Online]. Recuperado de: http://www.macval.fr/

Observatoire de l'art contemporaine.(2020). Sites Remarquables. observatoire-art-contemporain. Disponible en http://www.observatoire-art-contemporain.com/sites_remarquables/sites_remarquables.php?id=1009

Office du Tourisme et des Congrès. (2020). Les centres d'art de Paris, des lieux à découvrir. Parisinfo. Disponible en https:// www.parisinfo.com/decouvrir-paris/ guides-thematiques/art-contemporain-paris/les-hauts-lieux-de-l-art-contemporain/ les-centres-d-art-des-lieux-a-decouvrir?gclid=EAIaIQobChMInv3C697b6AIVDguRCh13sQUiEAAYAyAAEgLwBfD_BwE

Packer, J. \& Ballantyne, R. (2002). Motivational Factors and the Visitor Experience: A Comparison of Three Sites. Curator: The Museum Journal, 45(3), 183-198. https://doi.org/10.1111/j.2151-6952.2002. tb00055.x

Palais de Tokyo. (2020). Palais de Tokio. [Online]. Disponible en https://www. palaisdetokyo.com/

Platón. (1988). Diálogos. Madrid: Gredos.

Quetglas, J. (2004). Artículos de ocasión. Barcelona: Gustavo Gili.

Rancière, J. (2005). Sobre políticas estéticas. Barcelona: Museu d'Art Contemporani. 
Renault, M. (2000). Seuil du musée, deuil de la ville? La lettre de l'Ocim, 70, 15-20. Récupéré dans https://doc.ocim.fr/LO/LO071/ LO.71(3)-pp.15-20.pdf

République française. Ministère de la Culture. (2020). Centres d'art en Ile-de-France. culture.gouv.fr. Disponible en https://www. culture.gouv.fr/Sites-thematiques/Arts-plastiques/Structures-et-labels/Les-centres-dart-contemporain/Centres-d-art-en-Ile-deFrance

Rispal, A. (2009). L'architecture et la muséographie comme médiation sensible. Muséologies, 3(2), 90-101. https://doi. org/10.7202/1033564ar

Touraine, A. (2000). ¿Podremos vivir juntos? La Sociedad Multicultural. México, D.F.: Fondo de Cultura Económica.
Van Gennep, A. (1909). Les Rites de passage. Paris: E. Nourry.

Viatte, G. (2007). Le Centre Pompidou. Les années Beaubourg. Paris: Gallimard.

* Este artículo forma parte del proyecto de investigación FONDECYT N¹1170140: "EL LUGAR DEL ARTE CONTEMPORÁNEO EN SANTIAGO DE CHILE. Análisis de obras Emblemáticas de arquitectura publica y su vinculación con los habitantes desde la dimensión histórica, arquitectónica, urbana y sociocultural", financiado por el Fondo Nacional de Desarrollo Científico y Tecnológico de Chile.

Laura Gallardo Frías es Doctora en Arquitectura y Urbanismo por la Universidad Politécnica de Madrid. Arquitecta por la Universidad Politécnica de Cataluña. http://orcid.org/0000-00034814-3425

Paulina Román Manzo es Magister Internacional en Comunicación por la Universidad Diego Portales. Comunicadora Social por el Instituto AIEP, Santiago de Chile. 